\title{
Preliminary Abundance Analysis of Galactic Bulge Main Sequence, Subgiant, and Giant Branch Stars Observed During Microlensing with Keck/HIRES*
}

\author{
R. M. Cavallo ${ }^{a}$, K. H. Cook ${ }^{a}$, D. Minniti ${ }^{b}$, and T. Vandehei ${ }^{c}$ \\ ${ }^{a}$ Lawrence Livermore National Laboratory, 7000 East Ave, L-413, Livermore, CA 94550 \\ ${ }^{b}$ Universidad de Católica, Chile \\ ${ }^{c}$ University of California San Diego, San Diego, CA
}

\begin{abstract}
We present an abundance analysis of six main sequence turnoff, subgiant, and giant branch stars toward the Galactic bulge that were observed with Keck/HIRES during microlensing events. This is an early look at the first detailed chemical analysis of main sequence stars in the Galactic bulge. Lensing events allow the effective aperture of Keck to be increased beyond its current dimensions; although, some events still stretched its spectroscopic capabilities. Future large telescopes with high resolution and high throughput spectrometers will allow the study of abundances in distant stellar populations and in less evolved stars with greater ease.
\end{abstract}

Keywords: MACHO Project, Bulge, Abundances, Galactic Chemical Evolution

\section{INTRODUCTION}

The distance to the Galactic bulge has until recently kept the prospects of observing directly the chemical properties of its constituent stars just past the edge of possibility. Even now, with large-aperture telescopes such as the Kecks, the VLT, the HET, and the Gemini twins among others, only the brightest giants in the Bulge have been observable. ${ }^{1}$ A novel approach employed by the MACHO microlensing team has taken advantage of the temporary brightening of the source star during the lensing event to effectively decrease that once prohibitive distance to the Galactic center and allow for the observation of less evolved stars. For example, Minniti et al. ${ }^{2}$ first published results in 1997 on the presence of a Li I feature observed in a hot $\left(T_{\text {eff }}=6000 \mathrm{~K}\right)$ main sequence turn-off star. In this paper we discuss the full analysis of the that spectrum along with the spectra of five other stars observed during lensing events between 1997 and 1999. The results to date are still preliminary; however, they offer a glimpse into the diverse chemical evolution scenarios that can be found in and around the center of the Milky Way. Future telescopes with effective apertures in the 30-m or more range will make such observations commonplace rather than rare.

\section{OBSERVATIONS}

Between August 1997 and July 1999 we observed with the Keck I telescope and the HIRES echelle spectrometer 18 microlensing events that occurred when a small compact object (e.g., a normal star) crossed the line of sight between earth and a Bulge member, causing a temporary increase in the brightness of the latter by gravitational lensing. Of these, only six spectra had a sufficiently high signal-to-noise ratio to undergo a complete analysis that allowed us to determine: 1) radial velocities; 2) atmospheric parameters; and 3) chemical abundances. These six stars are described in Table 1, where they are identified by their lensing event number and their MACHO id in the first two columns, their unlensed magnitude and color in the next two columns, the epoch of their observation and the exposure time in the fifth and sixth columns, and an estimate of the signal-to-noise ratio in the last column. The $(V-R)_{0}$ colors are corrected based on recent extinction maps by Popowski,

This work was performed under the auspices of the U.S. Department of Energy, National Nuclear Security Administration by the University of California, Lawrence Livermore National Laboratory under contract No. W-7405-Eng-48.

Further author information: (Send correspondence to R.M.C.)

R.M.C.: E-mail: rcavallo@llnl.gov, Telephone 19254223277 
Cook, and Becker. ${ }^{3}$ We note that the events 97-BLG-45 and 97-BLG-47 were indeed the same star identified separately in overlapping MACHO fields. The event 98-BLG-6 was observed spectroscopically during two separate epochs in 1998, with the spectra being combined after ensuring no variation in the relative quality of each set of observations. The signal-to-noise ratio of this event represents that of the combined $(16,200 \mathrm{~s})$ spectra. Information regarding these and other microlensing events can be found on the MACHO project home page at http://www.macho.mcmaster.ca/.

Table 1. Program star data

\begin{tabular}{|l|c|c|c|c|r|c|}
\hline Lensing Event & F.T.S & $V$ & $(V-R)_{0}$ & Epoch & $t_{\exp }(\mathrm{s})$ & $\mathrm{S} / \mathrm{N}$ \\
\hline 97-BLG-45/47 & $142.27650 .6057 / 136.27650 .2370$ & 19.8 & 0.42 & 28 Aug 1997 & 10800 & 40 \\
\hline 97-BLG-56 & 403.47671 .57 & 16.1 & 0.85 & 27 Aug 1997 & 450 & 80 \\
\hline 98-BLG-6 & 402.48103 .1719 & 19.5 & 0.59 & 20 June 1998 & 10800 & 60 \\
\hline & & & & 18 Aug 1998 & 5400 & \\
\hline 99-BLG-1 & 121.22423 .1032 & 18.8 & 0.58 & 6 July 1999 & 7200 & 65 \\
\hline 99-BLG-8 & 403.47849 .756 & 15.8 & 0.82 & 6 July 1999 & 1800 & 130 \\
\hline 99-BLG-22 & 109.20893 .3423 & 19.6 & 0.79 & 7 July 1999 & 2700 & 90 \\
\hline
\end{tabular}

Our setup provided us with wavelength coverage from $4780 \AA$ to $7150 \AA$ with gaps between the orders that increased with wavelength. This allowed for the observation of many atomic absorption lines; however, it missed the important [O I] feature at $\lambda 6300$. We binned the data $2 \times 2$ in order to increase signal and decrease readout times. Combined with typical seeing of $1^{\prime \prime}$, this produced a resolution of $\mathrm{R} \sim 29,000$; sufficient for doing abundances work.

The data were reduced using the batch mode of MAKEE, written by T. Barlow and modified by him to accommodate our $2 \times 2$ binning format. MAKEE takes raw images as input and, after correcting for bias and flat fielding, extracts and wavelength calibrates the spectra. We then converted the MAKEE fits output files into an IRAF readable format using the MAKEE routine linear. Once the spectra were extracted we used the spectrum of a rapidly rotating hot star to correct for telluric lines by dividing the object spectrum by that of the rapid rotator, with the two spectra scaled and shifted by hand in the IRAF routine telluric. This removed most of the blaze function, allowing us to fit a low order polynomial to the data to produce flat, normalized spectra. We then measured the equivalent widths of lines by fitting gaussians with the IRAF routine splot. To test the quality of the data reduction, we reduced the spectra of many comparison stars that were taken with our program stars and that have equivalent widths published in the literature (e.g., Edvardsson et al. ${ }^{4}$ ). We found the agreement between our equivalent widths and the published data to be generally excellent, as shown in Fig. 1 for a sample star.

\section{ANALYSIS}

We began our analysis by determining heliocentric-corrected radial velocities. To do this we used the IRAF routine rvidlines and the iron line list of Nave et al. ${ }^{5}$ from which we selected approximately $450 \mathrm{Fe}$ I lines with accurate wavelengths that fell within our spectral coverage. During each epoch of observations we observed comparison stars with well determined radial velocities, and our analysis of the data showed excellent agreement between the radial velocities determined for these and their published values. The final column of Table 2 lists the radial velocities of our program stars.

Using the stellar atmosphere code MARCS (Gustafsson et al. ${ }^{6}$ ) and the LTE abundance analysis code MOOG $\left(\right.$ Sneden $\left.^{7}\right)$, we determined model atmosphere parameters for our program stars by using the Fe I and Fe II lines to achieve: 1) no trend in abundance versus excitation potential to determine $\mathrm{T}_{\text {eff }} ; 2$ ) no trend in abundance versus reduced width to determine microturbulence $(\xi)$; and 3) agreement in abundances from neutral and 


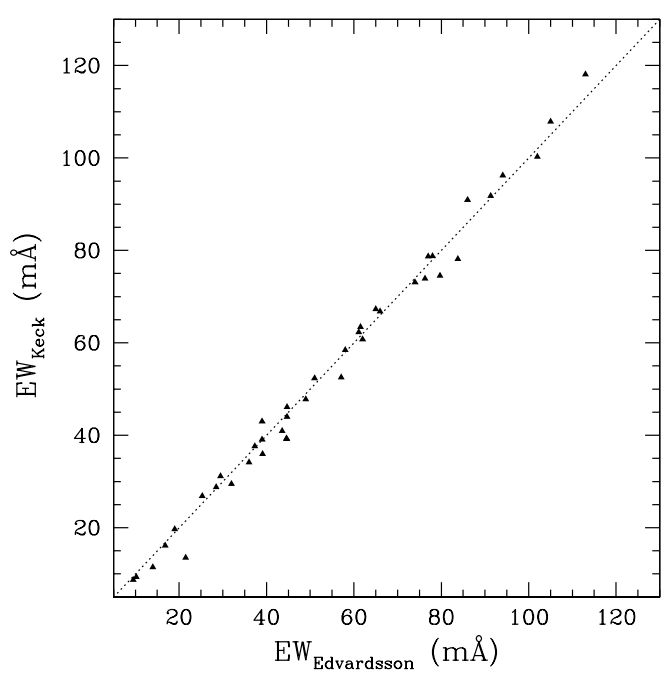

Figure 1. Equivalent width comparison between our data and Edvardsson et al. ${ }^{4}$ for the star HR 5019 . The dotted line represents perfect agreement.

ionized atoms to determine $\log g$. We used $\sim 49 \mathrm{Fe}$ I lines from Nave et al. ${ }^{5}$ and $\sim 14 \mathrm{Fe}$ II lines from Biémont et al., ${ }^{8}$ with the latter's $\log g f$ values corrected to $\log \epsilon(\mathrm{Fe})=7.52$ (a scale relative to hydrogen where $\log (\mathrm{H})$ is taken to be 12.00). The estimated uncertainties are $\pm 200 \mathrm{~K}$ in $T_{\text {eff }}, \pm 0.5$ dex in $\log g$, and $\pm 0.2 \mathrm{~km} \mathrm{~s}^{-1}$ in $\xi$. The derived atmospheric parameters are listed in Table 2.

We could have used the $V-R$ colors and the scales of Alonso et al. ${ }^{9}$ or Houdashelt, Bell, and Sweigart ${ }^{10}$ plus some assumptions about the distance modulus of each star to determine atmospheric parameters, but the uncertainties in the reddening are too large for this approach to be any more certain than using the iron lines.

Column 5 of Table 2 lists the derived MK classifications, which were found using line-depth ratios (e.g., Strassmeier and Fekel ${ }^{11}$ or Gray and Johanson ${ }^{12}$ ) and are not from the derived atmospheric parameters; however, it should be noted that in general the agreement between the physical parameters and the classification is quite good.

Table 2. Derived stellar parameters

\begin{tabular}{|l|c|c|c|c|l|}
\hline Lensing Event & $T_{\text {eff }}(\mathrm{K})$ & $\log g\left(\mathrm{~cm} \mathrm{~s}^{-1}\right)$ & $\xi\left(\mathrm{km} \mathrm{s}^{-1}\right)$ & MK class & $\mathrm{V}_{\text {rad }}\left(\mathrm{km} \mathrm{s}^{-1}\right)$ \\
\hline 97-BLG-45/47 & 6000 & 4.30 & 2.48 & G5 V & $+59.98 \pm 2.24$ \\
\hline 97-BLG-56 & 4480 & 1.40 & 1.84 & G8 III & $+177.0 \pm 1.9$ \\
\hline 98-BLG-6 & 5200 & 3.50 & 2.28 & G8 IV & $-64.24 \pm 1.68$ \\
\hline 99-BLG-1 & 5600 & 4.65 & 3.30 & G2 V & $+64.10 \pm 1.78$ \\
\hline 99-BLG-8 & 4150 & 0.95 & 1.91 & K2 III & $+194.9 \pm 1.7$ \\
\hline 99-BLG-22 & 5885 & 4.30 & 1.52 & F0 V & $+37.35 \pm 2.06$ \\
\hline
\end{tabular}

\section{RESULTS}

In the Tables 3-6 we present an early look into the abundances of our program stars, with the caveat that some numbers are subject to change; i.e., elements that suffer from heavy hyperfine splitting (hfs) and/or 
isotopic shifts will likely be revised in future work. ${ }^{13}$ Currently, we correct for such effects when possible by correcting one or two lines with the atomic data of Prochaska ${ }^{14}$ and applying an estimated correction to the total abundance. Abundances listed with a subscript (e.g., $[\mathrm{Sc} / \mathrm{Fe}]_{\mathrm{I}}$ and $[\mathrm{Sc} / \mathrm{Fe}]_{\mathrm{II}}$ ) are derived from both the neutral lines (I) and the singly ionized lines (II), and are listed separately. In all other cases the abundances come from lines of either ionization state.

Tables 3-6 do not list uncertainties for abundances other than iron; typically errors from line-to-line scatter for a given element are $\sim 0.2$ dex but can vary quite a bit. This is especially true for silicon where the rms is more like 0.5 dex; however, $[\mathrm{Si} / \mathrm{Fe}]$ is determined from lines with excitation potential around 6 e.V., while atmospheric parameters came from Fe I lines with $\chi<5$ e.V.. On the other hand $[\mathrm{Al} / \mathrm{Fe}]$ and $[\mathrm{Na} / \mathrm{Fe}]$ often showed good agreement between the abundances derived from different lines ( $\sigma<0.1$ dex).

We discuss the quality of the data for each star individually in Section 5.

Table 3. Derived abundances

\begin{tabular}{|l|c|c|c|c|c|c|c|c|}
\hline Lensing Event & {$[\mathrm{Fe} / \mathrm{H}]$} & {$[\mathrm{Na} / \mathrm{Fe}]$} & {$[\mathrm{Mg} / \mathrm{Fe}]$} & {$[\mathrm{Al} / \mathrm{Fe}]$} & {$[\mathrm{Si} / \mathrm{Fe}]$} & {$[\mathrm{Ca} / \mathrm{Fe}]$} & {$[\mathrm{Sc} / \mathrm{Fe}]_{\mathrm{I}}$} & {$[\mathrm{Sc} / \mathrm{Fe}]_{\mathrm{II}}$} \\
\hline 97-BLG-45/47 & $-0.28 \pm 0.22$ & +0.42 & +0.33 & +0.35 & +0.23 & +0.07 & $\ldots$ & -0.96 \\
\hline 97-BLG-56 & $-1.13 \pm 0.20$ & -0.03 & +0.79 & +0.15 & +0.30 & +0.24 & +0.65 & $\ldots$ \\
\hline 98-BLG-6 & $-0.22 \pm 0.18$ & +0.30 & +0.29 & +0.10 & +0.28 & -0.06 & +0.31 & $\ldots$ \\
\hline 99-BLG-1 & $-0.01 \pm 0.28$ & +0.67 & +0.81 & +0.67 & +0.33 & +0.13 & +1.36 & -0.12 \\
\hline 99-BLG-8 & $-1.12 \pm 0.20$ & +0.21 & +0.62 & +0.40 & +0.42 & +0.09 & +0.63 & +1.14 \\
\hline 99-BLG-22 & $-0.35 \pm 0.20$ & +0.12 & +0.59 & +0.33 & +0.34 & +0.20 & $\ldots$ & -0.45 \\
\hline
\end{tabular}

Table 4. Derived abundances (continued)

\begin{tabular}{|l|c|c|c|c|c|c|c|c|}
\hline Lensing Event & {$[\mathrm{Ti} / \mathrm{Fe}]_{\mathrm{I}}$} & {$[\mathrm{Ti} / \mathrm{Fe}]_{\mathrm{II}}$} & {$[\mathrm{V} / \mathrm{Fe}]_{\mathrm{I}}$} & {$[\mathrm{V} / \mathrm{Fe}]_{\mathrm{II}}$} & {$[\mathrm{Cr} / \mathrm{Fe}]_{\mathrm{I}}$} & {$[\mathrm{Cr} / \mathrm{Fe}]_{\mathrm{II}}$} & {$[\mathrm{Mn} / \mathrm{Fe}]$} & {$[\mathrm{Co} / \mathrm{Fe}]$} \\
\hline 97-BLG-45/47 & +0.92 & +0.50 & +0.52 & +1.23 & +0.45 & $\ldots$ & -0.25 & +0.61 \\
\hline 97-BLG-56 & +0.67 & +0.83 & +0.61 & $\ldots$ & +0.45 & +0.14 & -0.25 & -0.01 \\
\hline 98-BLG-6 & +0.50 & +0.43 & +0.54 & $\ldots$ & +0.08 & $\ldots$ & +0.08 & +0.00 \\
\hline 99-BLG-1 & +1.19 & +0.39 & +1.33 & +1.11 & +0.55 & $\ldots$ & +0.81 & +0.46 \\
\hline 99-BLG-8 & +0.68 & +0.34 & +0.17 & +0.48 & +0.35 & +0.75 & -0.28 & -0.15 \\
\hline 99-BLG-22 & +0.70 & +0.47 & +0.52 & +1.47 & +0.01 & $\ldots$ & -0.27 & +0.60 \\
\hline
\end{tabular}

\section{SUMMARY OF RESULTS}

\subsection{7-BLG-56 and 99-BLG-8}

We begin our discussion of the program stars with the cool giants. Both spectra have excellent signal-tonoise ratios, which comes as no surprise since these stars are already quite bright and being aided by lensing makes them that much brighter. They both contain the lowest $[\mathrm{Fe} / \mathrm{H}]$ in the sample, raising the possibility of systematic errors in their temperatures affecting the iron abundance determinations. We doubt this is the case since our reduction techniques have been compared favorably with our so-called "standard stars" and our model parameters match well with the MK classifications derived via line-depth ratios. The radial velocities of these two stars are the highest in the sample, giving rise to speculation that they could be members of the Sagittarius dwarf spheroidal; however, using the spectral type to estimate the absolute magnitudes and some assumptions about reddening, ${ }^{3}$ we derive distances to these stars of roughly $3.3-3.9 \mathrm{kpc}$. Compared with the 
Table 5. Derived abundances (continued)

\begin{tabular}{|l|c|c|c|c|c|c|c|c|c|}
\hline Lensing Event & {$[\mathrm{Ni} / \mathrm{Fe}]$} & {$[\mathrm{Zn} / \mathrm{Fe}]$} & {$[\mathrm{Y} / \mathrm{Fe}]_{\mathrm{I}}$} & {$[\mathrm{Y} / \mathrm{Fe}]_{\mathrm{II}}$} & {$[\mathrm{Zr} / \mathrm{Fe}]$} & {$[\mathrm{Nb} / \mathrm{Fe}]$} & {$[\mathrm{Mo} / \mathrm{Fe}]$} & {$[\mathrm{Ru} / \mathrm{Fe}]$} & {$[\mathrm{Ba} / \mathrm{Fe}]$} \\
\hline 97-BLG-45/47 & +0.28 & +0.29 & $\ldots$ & +0.35 & $\ldots$ & $\ldots$ & $\ldots$ & $\ldots$ & +0.39 \\
\hline 97-BLG-56 & +0.25 & $\ldots$ & +0.84 & +0.42 & +1.14 & $\ldots$ & +1.14 & +0.92 & +0.57 \\
\hline 98-BLG-6 & +0.19 & +0.48 & +0.92 & +0.91 & +0.83 & $\ldots$ & $\ldots$ & $\ldots$ & +0.66 \\
\hline 99-BLG-1 & +0.31 & -0.22 & +1.43 & +0.96 & +1.76 & $\ldots$ & +1.31 & +1.94 & +0.01 \\
\hline 99-BLG-8 & +0.12 & -0.03 & +0.76 & +0.61 & +1.15 & +1.54 & +0.92 & +1.11 & +0.68 \\
\hline 99-BLG-22 & +0.23 & +0.03 & $\ldots$ & +0.05 & $\ldots$ & $\ldots$ & $\ldots$ & $\ldots$ & +0.26 \\
\hline
\end{tabular}

Table 6. Derived abundances (continued)

\begin{tabular}{|l|c|c|c|c|c|}
\hline Lensing Event & {$[\mathrm{La} / \mathrm{Fe}]$} & {$[\mathrm{Ce} / \mathrm{Fe}]$} & {$[\mathrm{Pr} / \mathrm{Fe}]$} & {$[\mathrm{Nd} / \mathrm{Fe}]$} & {$[\mathrm{Eu} / \mathrm{Fe}]$} \\
\hline 97-BLG-45/47 & +0.97 & $\ldots$ & $\ldots$ & +1.72 & $\ldots$ \\
\hline 97-BLG-56 & +0.81 & +1.27 & +0.90 & +0.99 & +0.53 \\
\hline 98-BLG-6 & +0.99 & +1.10 & +0.83 & +0.92 & +1.09 \\
\hline 99-BLG-1 & +1.47 & +1.55 & $\ldots$ & +1.31 & +1.46 \\
\hline 99-BLG-8 & +0.91 & +1.11 & +0.94 & +0.85 & +1.34 \\
\hline 99-BLG-22 & $\ldots$ & +2.39 & +0.89 & +0.89 & $\ldots$ \\
\hline
\end{tabular}

Sagittarius dwarf spheroidal, which has a distance of $\sim 28 \mathrm{kpc},{ }^{15}$ these two stars clearly belong to a separate stellar population, even when accounting for errors in reddening or spectral type.

The abundance trends in both stars are consistent: the low- $\mathrm{Z}(\mathrm{Z}<40)$ elements are enhanced by about 0.5 dex while the high-Z elements are up by around $1 \mathrm{dex}$, indicating enhancements of $s$-process from possible AGB stars. The elements with estimated hfs corrections are $\mathrm{V}, \mathrm{Mn}$, and Co for both stars, leaving the abundances from the high-Z elements perhaps overestimated; although, not all of these require hfs corrections.

\subsection{8-BLG-6}

98-BLG-6 is roughly solar metallicity, particularly when the error bars are taken into account. The luminosity class is estimated from $\log g$ as either a main sequence turnoff star or a subgiant. The gravity does seem to be well determined given the excellent agreement between the abundances from the neutral and ionized atoms for the various species when available. The effective temperature/log $g$ combination along with the spectral type from line ratios is consistent with this star being a subgiant (see, e.g., Thévenin and Jasniewicz ${ }^{16}$ ), making 98-BLG-6 the only one in this study. The radial velocity for this star differs from the previous two by $250 \mathrm{~km} \mathrm{~s}^{-1}$ and is the only one of the six that is negative. Estimated hfs corrections were done for $\mathrm{V}$, Mn, and Co with corrections amounting to -0.65 dex for $[\mathrm{Mn} / \mathrm{Fe}]$ and -0.45 dex for $[\mathrm{Co} / \mathrm{Fe}]$. Given these large corrections for the iron-peak elements, the high- $Z$ elemental abundances should be viewed with caution. Finally, it should be noted that we are able to determine the abundances of 22 elements in a $V=19.5$ star only because of microlensing. Such detailed calculations are the exception at this magnitude, but should become trivial with a new age of $30-\mathrm{m}$ and larger class telescopes, provided they are outfitted with the proper instrumentation and can function in the visible bands.

\subsection{9-BLG-1}

According to our spectral typing, 99-BLG-1 is a solar analog in the Galactic bulge. Our derived physical parameters are also consistent with solar-like parameters within their errors. Some lines from Sc II, V I, Mn I, 
and Co I have been corrected for hfs effects. Both $[\mathrm{Sc} / \mathrm{Fe}]_{\mathrm{II}}$ and $[\mathrm{V} / \mathrm{Fe}]_{\mathrm{II}}$ change very little as a result, which is surprising given the large overabundance of $[\mathrm{V} / \mathrm{Fe}]_{\mathrm{II}}$; however, there are 18 other $\mathrm{V} \mathrm{II} \mathrm{lines} \mathrm{that} \mathrm{have} \mathrm{yet} \mathrm{to}$ be corrected and it remains to be seen whether the one line we corrected at $\lambda 5671$ is anomalous or is actually indicative of large $\mathrm{V}$ enhancements. The high- $\mathrm{Z}$ elements as a whole show the largest enhancements of all the stars. Ruthenium is particularly enhanced; however, the abundance comes from only one line at $\lambda 5636$ and this element does have seven stable isotopes with a rather flat distribution in relative abundance in nature among them. Barium is the one anomalous high-Z element in this star with a scaled solar ratio; this despite it's sister element, La, being enhanced by a factor of 30 relative to the sun. No hfs corrections have been applied to either of these elements. On the other hand, europium is enhanced and the high $\mathrm{Eu} / \mathrm{Ba}$ ratio would indicate heavy $r$-process influences. Further analysis of this star should yield an interesting chemical history.

\subsection{9-BLG-22}

This star is close to being a solar analog in temperature and gravity; however, the derived spectral type appears to make it too hot. The signal-to-noise ratio is quite good for this star (especially considering it has an unlensed $V$ magnitude of 19.6), giving us confidence in our spectral analysis. Relatively little information is available for the high-Z elements, but once again, $[\mathrm{Ba} / \mathrm{Fe}]$ is near solar while the heavier elements remain high. $[\mathrm{Sc} / \mathrm{Fe}]_{\mathrm{II}}$, $[\mathrm{V} / \mathrm{Fe}]_{\mathrm{I}}$, and $[\mathrm{Mn} / \mathrm{Fe}]_{\mathrm{I}}$ have estimated corrections for hfs effects. Despite the quality of the spectrum, abundances of $\mathrm{Zn}, \mathrm{Y}, \mathrm{Ce}, \mathrm{Pr}$, and $\mathrm{Nd}$ come from only one or two lines each, which is atypical compared with the brighter stars.

\subsection{7-BLG-45/47}

We conclude our summary with a discussion of 97-BLG-45/47. Like 99-BLG-22, the derived stellar parameters are at odds with the spectral type, and given the poor signal-to-noise ratio in the spectrum it is difficult to decide on a preferred set of parameters. The choices we adopted in Table 2 are found through spectroscopic means and are consistent with the parameters listed in Minniti et al., ${ }^{2}$ making the star a G0 dwarf. 97-BLG-45/47 is slightly underabundant in iron and shows a range of scatter in the remaining elements. $[\mathrm{Nd} / \mathrm{Fe}]$ is particularly high, but the results do come from five lines and show only 0.34 dex scatter. The results for $[\mathrm{Sc} / \mathrm{Fe}]_{\mathrm{II}},[\mathrm{V} / \mathrm{Fe}]_{\mathrm{I}}$, and $[\mathrm{Mn} / \mathrm{Fe}]_{\mathrm{I}}$ do include estimated hfs corrections.

We revisited the $\lambda 6708 \mathrm{Li}$ I feature in the spectrum of $97-\mathrm{BLG}-45 / 47$ as first studied by Minniti et al.. ${ }^{2}$ They measured an equivalent width of $47 \mathrm{~m} \AA$ and estimated an abundance of $\log \epsilon(\mathrm{Li})=2.25$ from published tables. We measure the equivalent width to be $37.3 \mathrm{~m} \AA$, which when run in a full synthesis using the line list from Ford et al., ${ }^{17}$ results in $\log \epsilon(\mathrm{Li})=2.25$; i.e., good agreement with the earlier work. A question, however, arises as to the validity of the detection, given the noise in the spectrum and the fact that the center of the measured line is $0.12 \AA$ to the red of the strongest feature in the Li doublet, while the center of the Fe I line at $\lambda 6705$ is well matched in the data, as can be seen in Fig. 2.

Finally, we note that our radial velocity is $30 \mathrm{~km} \mathrm{~s}^{-1}$ lower than that listed in Minniti et al. ${ }^{2}$ for uncertain reasons. Given the excellent agreement we obtained between the radial velocities of our "standards" observed on the same nights as $97-B L G-45 / 47$ and their published values, we are quite confident in our determination listed in Table 2.

\section{CONCLUSIONS}

Microlensing has allowed us to derive a reasonably large and detailed set of abundances for a group of stars that have heretofore been beyond our capabilities. While some work on the details remains to be done before conclusions can be firmly drawn, we have been able to study abundance trends in stars as diverse as solar analogs and metal-poor red giants. Our early results show a trend of overabundance in nearly all the elements regardless of metallicity or spectral class. The two giants in the study also have the lowest metallicities and the highest radial velocities, and they are easily ruled out as members of the Sagittarius dwarf spheroidal. We have also re-analyzed the Li abundance in the star 97-BLG-45 and have found that we cannot conclusively rule for or against the presence of Li. All the spectra show some hint of Li $\lambda 6708$ and we will examine these in kind to determine abundances or upper limits. 


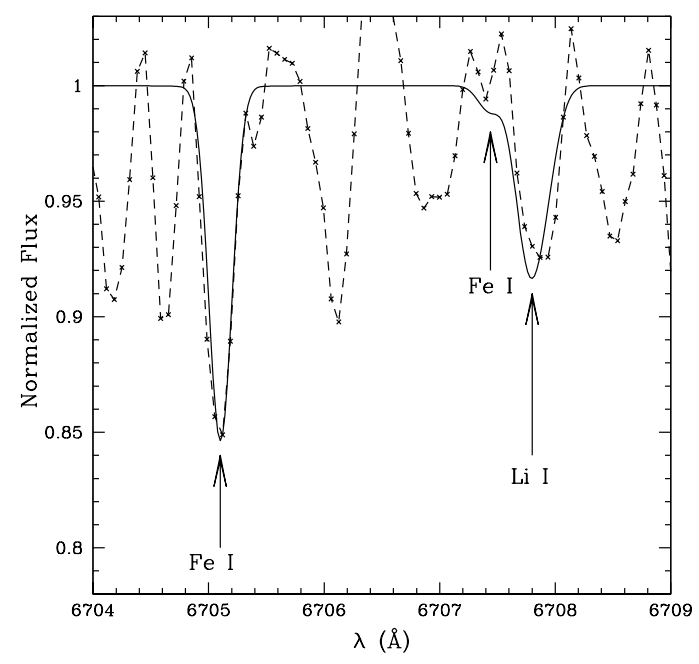

Figure 2. The region around the Li I doublet at $\lambda 6708$ showing the spectrum of 97-BLG-45/47 (x's and dotted line) and the synthetic spectrum with $\log \epsilon(\mathrm{Li})=2.25$ and $\log \epsilon(\mathrm{Fe})=7.42$ (solid line).

Our results are likely to change for some of the elements where hfs or isotopic corrections are still necessary. When possible we corrected for hfs effects by correcting one line and applying a shift to the results from all the lines. Sometimes the shift was large $(>0.5 \mathrm{dex})$ and sometimes it was nonexistent.

Thanks to a helping hand from Nature, we were able to get a glimpse at the functioning of Keck as a much larger telescope than its current 10-m diameter. Future 30-m and larger class telescopes will make such observations commonplace and allow us to learn truly the diverse histories of the Galactic bulge stellar population.

\section{ACKNOWLEDGMENTS}

We wish to thank Stefan Keller for helping us classify our stars, Piotr Popowski for providing us with extinction maps of the MACHO fields, and Tom Barlow for modifying his MAKEE routine to accept our data format and answering our many questions. This research has made use of the SIMBAD database, operated at CDS, Strasbourg, France.

\section{REFERENCES}

1. R. M. Rich and A. McWilliam, "Abundances of Stars in the Galactic Bulge Obtained Using the Keck Telescope," J. Bergeron. ed., Proc. SPIE 4005, pp. 150-161, 2000.

2. D. Minniti, T. Vandehei, K. H. Cook, K. Griest, and C. Alcock, "Detection of Lithium in a Main-Sequence Bulge Star Using Keck I as a 15 Meter Diameter Telescope," ApJ 499, pp. L175-L178, 1998.

3. P. Popowski, K. Cook, and A. Becker, "The Large-Scale Extinction Map of the Galactic Bulge from the MACHO Project Photometry," in prep..

4. B. Edvardsson, J. Andersen, B. Gustafsson, D. L. Lambert, P. E. Nissen, and J. Tomkin, "The Chemical Evolution of the Galactic Disk - Part 2 - Observational Data," A $\& A S$ 102, pp. 603-605, 1993.

5. G. Nave, S. Johanson, R. C. M. Learner, A. P. Thorne, and J. W. Brault, "A New Multiplet Table for Fe I," ApJSS 94, pp. 221-459, 1994.

6. B. Gustafsson, R. A. Bell, K. Eriksson, and Å. Nordlund, "A Grid of Model Atmospheres for Metal-deficient Giant Stars. I," A $\& A$ 42, pp. 407-432, 1975. 
7. C. Sneden, "The Nitrogen Abundance of the Very Metal-poor Star HD 122563," ApJ 184, pp. 839-849, 1973.

8. E. Biémont, M. Baudoux, R. L. Kurucz, W. Ansbacher, and E. H. Pinnington, "The Solar Abundance of Iron - A 'Final' Word," A\&A 249, pp. 539-544, 1991.

9. A. Alonso, S.Arribas, and C. Martínez-Roger, "The Effective Temperature Scale of Giant Stars (F0-K5). II. Empirical Calibration of $T_{\text {eff }}$ Versus Colours and [Fe/H]," $A \& S S$ 140, pp. 261-277, 1999.

10. M. L. Houdashelt, R. A. Bell, and A. V. Sweigart, "Improved Color-Temperature Relations and Bolometric Corrections for Cool Stars," AJ 119, pp. 1448-1469, 2000.

11. K. G. Strassmeier and F. C. Fekel, "The Spectral Classification of Chromospherically Active Binary Stars with Composite Spectra," A $\mathscr{E} A 230$ pp. 389-404 1990.

12. D. F. Gray and H. L. Johanson, "Precise Measurement of Stellar Temperatures Using Line-depth Ratios," PASP 103, pp. 439-443, 1991.

13. R. M. Cavallo, K. H. Cook, D. Minniti, T. Vandehei, "Keck Observations of Abundances in Lensed Main Sequence, Subgiant, and Giant Stars in the Galactic Bulge," in prep..

14. J. X. Prochaska, S. A. Naumov, B. W. Carney, A. McWilliam, and A. M.Wolfe, "The Galactic Thick Disk Stellar Abundances," AJ 120, pp. 2513-2549, 2000.

15. A. Sarajedini and A. C. Layden, "A Photometric Study of the Globular Cluster M54 and the Sagittarius Dwarf Galaxy: Evidence for Three Distinct Populations," AJ 109, pp. 1086-1094, 1995.

16. F. Thévenin and G. Jasniewicz, "Barium-rich G stars in the nuclei of the planetary nebulae Abell 35 and LoTr5," A\&3A 320, pp. 913-919, 1997.

17. A. Ford, R. D. Jeffries, B. Smalley, S. G. Ryan, W. Aoki, S. Kawanomoto, D. J. James, and J. R. Barnes, "Lithium $6104 \AA$ in Population II Stars," $A \mathscr{E} A$ in press. 